\title{
15
}

\section{An Idea Once Crystalised}

\author{
Emma Purshouse
}

Maybe it was a weird thing to do to send a paper bag full of suck in the post, not so much as a note,

their smell permeating the jiffy bag waiting to waft out into your room, that heady mix of the medicinal

and the factory filling your air with the sort of smell you might put on a wound or a scratch where the swarf has bit.

The sort of smell that might, one cold night, remind you of home, of me, of the Black Country.

E. Purshouse $(\varangle)$

Wolverhampton, UK 